\title{
Explosive nucleosynthesis in tidal disruption events of massive white dwarfs, and their debris
}

\author{
Ataru Tanikawa ${ }^{1,2}$, Yushi Sato ${ }^{1,3}$, Ken'ichi Nomoto ${ }^{4,5}$, \\ Keiichi Maeda ${ }^{6,4}$, Naohito Nakasato ${ }^{7}$ and Izumi Hachisu ${ }^{1}$ \\ ${ }^{1}$ Department of Earth Science and Astronomy, College of Arts and Sciences, The University of \\ Tokyo, 3-8-1 Komaba, Meguro-ku, Tokyo 153-8902, Japan; tanikawa@ea.c.u-tokyo.ac.jp \\ ${ }^{2}$ RIKEN Advanced Institute for Computational Science, 7-1-26 Minatojima-minami-machi, \\ Chuo-ku, Kobe, Hyogo 650-0047, Japan \\ ${ }^{3}$ Department of Astronomy, Graduate School of Science, The University of Tokyo, 7-3-1 \\ Hongo, Bunkyo-ku, Tokyo 113-0033, Japan \\ ${ }^{4}$ Kavli Institute for the Physics and Mathematics of the Universe (WPI), The University of \\ Tokyo, 5-1-5 Kashiwanoha, Kashiwa, Chiba 277-8583, Japan \\ ${ }^{5}$ Hamamatsu Professor \\ ${ }^{6}$ Department of Astronomy, Kyoto University, Kitashirakawa-Oiwake-cho, Sakyo-ku, Kyoto \\ 606-8502, Japan \\ ${ }^{7}$ Department of Computer Science and Engineering, University of Aizu, Tsuruga Ikki-machi \\ Aizu-Wakamatsu, Fukushima 965-8580, Japan
}

\begin{abstract}
We perform SPH simulations coupled with nuclear reactions to follow tidal disruption events (TDEs) of white dwarfs (WDs) by intermediate mass black holes (IMBHs). We consider an oxygen-neon-magnesium $(\mathrm{ONeMg}) \mathrm{WD}$ with $1.2 M_{\odot}$ as well as a helium $(\mathrm{He}) \mathrm{WD}$ with $0.3 M_{\odot}$, and a carbon-oxygen $(\mathrm{CO}) \mathrm{WD}$ with $0.6 M_{\odot}$. Our WD models have different numbers of SPH particles, $N$, up to a few 10 million. We find that nucleosynthesis does not converge against $N$ even for $N>10^{7}$. For all the WDs, the amount of radioactive nuclei, such as ${ }^{56} \mathrm{Ni}$, decreases with increasing $N$. Nuclear reactions might be extinguished for infinitely large $N$. Our results show that these kinds of TDEs, if solely powered by radioactive decays, are much dimmer optical transients similar to Type Ia supernovae as previously suggested.
\end{abstract}

Keywords. hydrodynamics, nuclear reactions, nucleosynthesis, white dwarfs

\section{Introduction}

A number of tidal disruption events (TDEs) has been found. Some of them are possibly TDEs of a WD (WD TDEs). We focus on the WD TDE as an optical transient resulting from its thermonuclear explosion. In a WD TDE, the WD is heated by compression in the direction perpendicular to the orbital plane. Then, the WD may undergo explosive nuclear burning, yielding radioactive nuclei such as ${ }^{56} \mathrm{Ni}$. Rosswog et al. (2008) and Rosswog, Ramirez-Ruiz \& Hix (2009) studied WD TDEs and their nucleosynthesis signatures. A WD TDE needs not only an adiabatic compression but also a shock heating in order to reach high temperature enough to start explosive nuclear reactions. However it is not clear whether previous simulations have high enough resolution to detect the shock wave.

In this paper, we perform SPH simulations with various numbers of SPH particles from a few 0.01 million up to a few 10 million. We aim to assess whether the shock wave is generated, and whether nucleosynthesis is converging against $N$ even if the shock wave is not generated. 


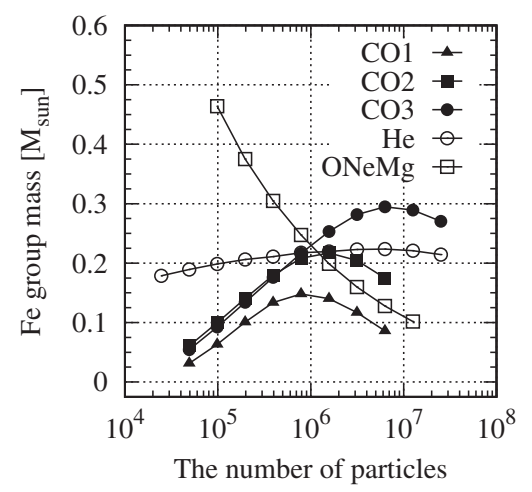

Figure 1. Mass fractions of Fe group elements as a function of $N$ at the time just after the nuclear reactions have ceased

\section{Method}

We follow the evolution of WD TDEs by means of our SPH code coupled with a nuclear reaction network. We adopt SPH modeling for the WDs, and use a fixed potential to model the IMBH gravity. Our SPH code solves the vanilla ice SPH equations with selfgravity among SPH particles with adaptive gravitational softening. We use the Helmholtz equation of state with (or without) Coulomb corrections (Timmes \& Swesty 2000), and include nuclear reactions with Aprox13 (Timmes, Hoffman, \& Woosley 2000).

We adopt a He WD with $0.3 M_{\odot}$, a $\mathrm{CO}$ WD with $0.6 M_{\odot}$, and a ONeMg WD with $1.2 M_{\odot}$. We set an IMBH with $500 M_{\odot}$ for the He WD and $\mathrm{CO}$ WD, and an IMBH with $100 M_{\odot}$ for the ONeMg WD. For the CO WD, we model the IMBH gravity as Newtonian potential, the Paczyński-Wiita (PW) potential and a generalized Newtonian potential obtained by Tejeda \& Rosswog (2013). The model names are "CO1", "CO2", and "CO3". For the He WD and ONeMg WD, we assume PW potential. These model names are "He" and "ONeMg". In all the models, we change the number of particles for the WDs, $N$, from a few 0.01 million up to a few 10 million. The ratios of pericenter distances to tidal radii are 5.0 for models COs and He, and 3.0 for model ONeMg.

\section{Results}

Figure 1 shows that the nucleosynthesis does not converge against $N$ even for $N>10^{7}$. For model $\mathrm{ONeMg}$, the amount of these elements decreases monotonically. For the other models, these elements also decrease at $N>10^{7}$. Nuclear reactions might be extinguished for infinitely large $N$. Our results show that these kinds of TDEs, if solely powered by radioactive decays, are much dimmer optical transients similar to Type Ia supernovae as previously suggested.

\section{References}

Rosswog, S., Ramirez-Ruiz, E., \& Hix, W. R. 2009, ApJ, 695, 404

Rosswog, S., Ramirez-Ruiz, E., Hix, W. R., \& Dan, M. 2008, Computer Physics Communications, 179,184

Tejeda, E., \& Rosswog, S. 2013, MNRAS, 433, 1930

Timmes, F. X., Hoffman, R. D., \& Woosley, S. E. 2000, ApJS, 129, 377

Timmes, F. X. \& Swesty, F. D. 2000, ApJS, 126, 501 